\title{
4 Kulturgeschichtliche Perspektive auf die Prävention psychischen Leidens
}

Die Gesundheit wird als gesellschaftliches Gut und individuelle Disposition in unterschiedlichen Zusammenhängen thematisiert. Aus kulturgeschichtlicher Perspektive lässt sich erkennen, welchen Stellenwert und welche Wandelformen die Gesundheit in den westlichen industriellen Gesellschaften erfahren und wie sie das gegenwärtige Verständnis vom gesunden oder kranken Leben geprägt hat. Im Folgenden werden drei Perspektiven aufgezeichnet, die die unterschiedlichen Aspekte des Regiert-Werdens durch Prävention diskutieren.

\subsection{Prävention als Kulturtechnik}

Die im Rahmen des Forschungsverbundes Präventives Selbst - Interdisziplinäre Untersuchung einer emergenten Lebensform entstandenen Beiträge liefern wichtige Erkenntnisse zur "Entwicklung moderner Praktiken der Gesundheitsprävention.« (Lengwiler \& Madarász 2010: 9) Zwar hat laut der Autoren des Bandes »das Thema der Gesundheitsprävention in den letzten Jahren politisch und gesellschaftlich an Bedeutung gewonnen« (a.a.O.: 12), doch ihre Geschichte sei »noch weitgehend ungeschrieben« (a.a.O.: 11). Diesem Zustand haftet gleichzeitig etwas Unwirkliches und Unauffälliges an:

»Unwirklich, weil Prävention einen Schaden antizipiert, der meist gar nicht eintritt, weil er vorsorglich verhindert werden soll. Und unauffällig, weil Prävention meist die Ebene unspektakulärer Alltagsverhaltens (Essen und Trinken, Rauchen, Bewegung etc.) betrifft und dabei oft in wenig sichtbaren Infrastrukturbereichen ansetzt, etwa bei der Nahrungsmittelhygiene, der Fabrikinspektion oder allgemein der Hygienisierung des öffentlichen Raumes.« (a.a.O.: 11f)

In den gesammelten Untersuchungen wird die Prävention als eine Kulturtechnik zum Gegenstand der Analyse gemacht. Als solche wurde nämlich Prävention schon im 19. Jahrhundert zum Leitmotiv »der sozialwissenschaftlichen Erkenntnisgewinnung und der davon ausgehenden sozialpolitischen Interventionen" (a.a.O.: 13) herausgestellt. In den späteren Theoriedebatten wurde sie dann laut Autoren als eine »auf rationalem Kalkül beruhende Zukunftsbeherrschung (a.a.O.: 14), wie bei Reinhart Kosseleck und Lucian Hölscher bzw. als eine »wissenschaftlich-technologisch fundierte, mit wirkungsmächtigen Rationalitätsansprüchen auftretende Form der Sozial- und Bevölkerungspolitik« (ebd.), wie bei Niklas Rose und Ulrich Bröckling (im Rückgriff auf Michel Foucault), gedacht.

Kulturgeschichtlich lassen sich auf der Ebene der Akteure vier Gruppen unterscheiden, bei welchen das präventive Verhalten untersucht werden kann. So war es erstens das Anliegen des Staates seine »Bevölkerung - als ökonomische und mili- 
tärische Ressource - möglichst effizient und nachhaltig zu mobilisieren.« (a.a.O.: 15) Sodann haben auch die privatwirtschaftlichen Akteure, wie etwa private Spitäler, Versicherungs- oder Pharmaindustrie, für »die Propagierung präventiver Verhaltensnormen und in Verbindung damit [für, J. Z.] eine `Ökonomisierungく der Präventionsdiskurse« (ebd. [Herv. i. O.]) gesorgt. Außerdem forderten die zivilgesellschaftlichen Vereinigungen, »etwa die Hygienebewegung des 19. Jahrhunderts oder alternativ-medizinische Kreise des 20. Jahrhunderts [...] eine stärker >lebensweltlichく verankerte[...] >Präventionskultur«.« (a.a.O.: 15f [Herv. i. O.]) Schließlich hat sich in der zweiten Hälfte des 20 . Jahrhunderts eine Subjektivierung der präventiven Verantwortung vollzogen, in der die individuellen Akteure bzw. »das >präventive Selbst<, jenes rationale, krankheitsminimierend agierende Subjekt« (a.a.O.: 16 [Herv. i. O.]) selbst für seine Gesundheit verantwortlich geworden ist. Diese Analyseeintrittspunkte ermöglichen es, die »Paradoxien präventiver Verhaltensdispositionen« (ebd.) aus verschiedenen Blickwinkeln zu beleuchten. So ließe sich einerseits der beobachtete »Widerspruch zwischen wissenschaftlichem Präventionsanspruch und alltäglichen Präventionspraktiken« (ebd.) erhellen, andererseits die durch den modernen Präventionsdiskurs entstandene »nicht-intendierte[...] Vermehrung von Unsicherheit« (ebd.) problematisieren.

Die hier pointierte Spannung zwischen den auf die Bevölkerung gerichteten wissenschaftlichen Präventionsmodellen sowie den individuellen alltagspraktischen Erfahrungen auf der einen Seite, und der äußeren (Umweltfaktoren, Lebensbedingungen) wie inneren (körperliche Zustände, Lebensstille) Natur der Präventionsmaßnahmen auf der anderen Seite umfangen den Bereich der Regierungstechnologien, wie sie von Michel Foucault gedeutet und beschrieben wurden (vgl. Foucault [1984] 2005b: 900) und bilden somit das Objekt analytischer Bemühungen der Gouvernementalitäts-Studien. Demnach entsteht hier die Frage, inwieweit die Einrichtung präventiver Maßnahmen das alltägliche Verhalten prädisponiert oder aber wie die Problematisierung der Lebensbedingungen den individuellen Lebensstil prägt und anleitet. Die Führung zur Selbstführung wird in dem Ineinandergreifen des öffentlichen und privaten Lebens beobachtbar und analysierbar. Eben der Raum dieses Ineinandergreifens wird durch die Prävention als Kulturtechnik kolonisiert.

\subsection{Gesundheit als Zielscheibe der Prävention}

Aus geschichtlicher Perspektive lassen sich mehrere Versuche feststellen, wie die Gesundheit zur Zielscheibe präventiver, vorbeugender Maßnahmen erhoben wurde. Vor allem im 19. und 20. Jahrhundert avancierte allmählich die »Vorsorge von einem Projekt einzelner Schichten zu einer gesamtgesellschaftlichen Aufgabe.« (Thießen 2013: 354) Diese Vorsorge kristallisierte sich in dem Versuch, »in gesellschaftliche Prozesse wie Pauperismus, Industrialisierung und Städte- 
wachstum und die damit verbundenen gesundheitlichen Gefahren im Sinne einer Demokratisierung der Gesellschaft formend einzugreifen « (Stöckel \& Walter 2002: 11) und hygienische, prophylaktische und präventive Vorsorgemaßnahmen einzuleiten. Mit den verschiedenen Eingriffen in die Gesundheit des Einzelnen und der ganzen Gesellschaft entwickelten sich dann schrittweise Verfahren, »durch die die Menschen zu einem bestimmten gesundheitsbezogenen Verhalten angeleitet und Regierungen $\mathrm{zu}$ bestimmten gesundheitspolitischen Strategien motiviert und für diese legitimiert wurden." (Poczka 2017: 421) Dadurch konnten gesundheitspolitische Maßnahmen »auf den Schutz des ganzen >Volkskörpers`, auf die Verhütung von Gefahren für die Allgemeinheit sowie auf die Minimierung kollektiver Risiken im Dienste des >öffentlichen Interesses« abzielen (Thießen 2013: 354 [Herv. i. O.]) und gleichzeitig »Einfluss auf Alltagspraktiken, auf Vorstellungen und Formen der Lebens- und Familienplanung, auf den Umgang mit Gesundheit und Sexualität« nehmen (a.a.O.: 358). Diese umfassende Einwirkung präventiver Eingriffe in alle privaten wie öffentlichen Lebensbereiche entwickelte sich in dem Maße, wie die Prävention gesellschaftlich verankert und normalisiert wurde.

Der Normalisierung von Prävention hat ein paradigmatischer Wechsel geholfen, welcher sich im Zusammenhang mit der Umdeutung der Gesundheit durch die Weltgesundheitsorganisation (WHO) im Jahr 1946 vollzogen hat. Der durch die WHO neu generalisierte Gesundheitsbegriff definierte die Gesundheit »nicht mehr als Abwesenheit von Krankheit, sondern aufgrund positiver Qualitäten wie körperlichen, geistigen und sozialen Wohlbefindens« (Lengwiller \& Madarász 2010: 21), womit ein Grundstein »für eine schrittweise Auflösung der Grenze zwischen Gesundheit und Krankheit " gelegt wurde (ebd.). Indessen richtete sich auch die Prävention nicht mehr auf die Krankheitsverhinderung, sondern vor allem auf die Gesundheitssicherung (vgl. ebd.). ${ }^{13}$ Krankheit und Gesundheit wurden somit nicht nur neu problematisiert, sondern haben sich »als soziale und zugleich diskursive Konfliktfelder « (Poczka 2017: 17) in eine allgemeine Geschichte der Prävention integriert und die bisher nicht ausreichend beantwortete Frage nach ihrer Regierbarkeit aufs Neue gestellt: »Wie hat unsere Gesellschaft gelernt, zukünftige Erkrankungen, die noch nicht eingetroffen sind und von denen sich im Einzelfall nicht wissen lässt, ob sie jemals eintreten werden, als reale Probleme zu behandeln?« (Leanza 2017: 16) Damit hat die Prävention eine Arbeit an dem Virtuellen begonnen, die darauf abzielt, »das Werden in seiner Ereignishaftigkeit zu lenken, um drohenden

13 Das Anliegen, den Gesundheitsbegriff neu definieren zu müssen, entstammt aus der Sorge um das Wohlbefinden der Bevölkerungen in den Ländern der Dritten Welt und sollte zum »Teil eines globalisierten Gerechtigkeitsdenkens « (Lengwiller \& Madarász 2010: 21) werden. Welche Konsequenzen dies gebracht hat, verdeutlicht im Hinblick auf globale Biopolitik bspw. Susanne Schultz (vgl. Schultz 2011). 
Gefahren auszuweichen.«(a.a.O.: 12) Diese Arbeit am Virtuellen formiert das Zukünftige und konstituiert dessen mögliche Horizonte, weshalb sie "geschichtlich wandelbar und auf soziale Zusammenhänge angewiesen [ist], in denen sie angewandt, verbreitet und tradiert wird.«(a.a.O.: 19f) ${ }^{14}$ Zur Untersuchung der Prävention und ihrer zeitlichen Formen wurden einige Versuche bereits unternommen, die sich auf ihre Genealogie im Foucault'schen Sinne konzentriert (vgl. a.a.O.: 21) bzw. einen vergleichenden Blick auf ihre institutionelle wie gesellschaftliche Verankerung geworfen haben (vgl. Hähner-Rombach 2015). Offen und bisweilen unerforscht bleibt, welche konkreten Praktiken mit der Prävention entstanden sind und inwiefern sie zu einem »Modus der Selbstvergewisserung« geworden sind (Thießen 2013: 365).

\subsection{Ein kulturgeschichtlicher Blick auf Leiden und Unbehagen}

Die Wahrnehmung und Behandlung des Leidens gehört zu den Kernelementen jeder Kultur. Leidensausdrücke widerspiegeln nicht nur die Sensitivität für menschliche Erfahrungen, sondern bilden zugleich Kontrapunkte zu grundlegenden Werten und Normen, zu dem, was die Kultur für (überlebens-)wichtig hält. Diese Leidenserfahrungen lassen sich auf drei Ebenen unterscheiden - als individuelle, kollektive und globale Erfahrungen.

In der westlichen Kultur, geprägt neben vielen anderen auch von der jüdischchristlichen geistigen Tradition, wird das Leiden des Individuums in den alttestamentlichen Büchern und Psalmen umfangreich beschrieben. Ihre Mikroexegese zeigt, dass die niederdrückenden Gefühle einerseits durch Körperhaltung (hebr. qādar - niedergedrückt sein, schwarz werden, sich verfinstern - als Gegensatz zur Aufhellung von Antlitz Gottes; šhh (schachar) - sich bücken, beugen, niedergebeugt sein) und -Bewegung (hebr. ndd (nadad) - rastlos, ziellos fliehen, fern machen; nedudîm - Unrast, Schlaflosigkeit; rwd (rûd) - umherirren) ausgedrückt werden, andererseits sich in der (Un-)Fähigkeit äußern, Beziehungen aufzubauen und aufrechtzuerhalten (das Fliehen von und die Rückkehr zur Gemeinschaft umrahmen die Leidensgeschichte des Individuums im Alten Testament, etwa im Hiobbuch) (vgl. Janowski 2015). Gleichzeitig wird das Leiden in unterschiedlichen Bildern ausgedrückt - etwa mit dem Gesicht zwischen den Knien, was zugleich Konzentration und den Blick in sich selbst darstellt - oder in dem Spiel der Farben umschrieben - etwa Licht und Finsternis, sehr anschaulich in Julia Kristevas Bild der schwarzen Sonne enthalten (vgl. Kristeva [1987] 2007). Das moderne Individuum des 20. und 21. Jahrhunderts ist im Vergleich zum alttestamentlichen Menschen,

14 Besonders gut ließen sich die verschiedenen Ausdeutungen der Prävention an den geschlechtlichen Differenzierungen beobachten (vgl. Pfütsch 2017). 
der in den Psalmen sein Leiden äußert und es vor Gott als der höchsten Heilsinstanz ausbreitet, mit den leitenden Narrativen des selbsterzeugten Erfolgs und Glücks konfrontiert (vgl. Kleiner \& Suter 2018: 12). Ein Burnout-Leidender wird einerseits mit der Schattenseite gegenwärtiger Gesellschaft konfrontiert, »in der das selbstverschuldete Scheitern und der drohende soziale Abstieg überall lauern können« (a.a.O.: 10), andererseits "wird dieser Chronik individuellen Scheiterns eine gelingende Variante der Selbstfindung, ein erfolgreicher >pursuit of happiness hinzugefügt.« (ebd. [Herv. i. O.]) Diese doppelte Bewegung bewirkt eine Transformation in »eine Art stotales Subjekt«« (a.a.O.: 11 [Herv. i. O.]), das die Misere seines Lebens erkennen und die Potentiale seiner Optimierung aktivieren kann. Somit schwinden die Grenzen »zwischen Glück und Erfolg, Leistung und Versagen, Anpassung und Selbsterlösung« (a.a.O.: 12), denn jede Leidenserfahrung birgt in sich die Chance auf Neuentdeckung bisher unbekannter und nicht geforderter Fähigkeiten. Die neuen individuellen Pathologien markieren indessen »den Einsatzpunkt einer wirksamen Intervention von außen und einer gelingenden Selbstintervention« (ebd.), woran Therapie, Begleitung und Prävention ansetzen können. Dies geschieht vor dem Hintergrund einer Ratgeber-Industrie, die einerseits ein Psychoideal des modernen Individuums verkündet, das jedoch andererseits von den Ratgebern selbst nicht verkörpert werden kann (vgl. Lütz 2011: 16:57 min.)

Eine semantische Abgrenzung ließe sich auch auf die kollektive Wahrnehmung des Leidens übertragen. Hartmut Böhme unterscheidet in dieser Hinsicht zwei Achsen:

»die >vertikale Bewegung « von oben nach unten, der Gravitation folgend, worin die Schwere des Körpers leitend wird und vom Lebensgefühl temporär (episodisch) oder dauerhaft (rezidivierend) Besitz ergreift - im Gegensatz zu jenen leiblichen, ebenfalls vertikal ausgerichteten Gestimmtheiten, in denen Fröhlichkeit, Liebe, Erhabenheit, Ekstase anti-grav wirken, nämlich Cefühle des Leichten, aufwärts Drängenden, ja Schwerelosen wecken. Der Ermüdete oder Erschöpfte spürt dagegen ein unwiderstehliches Niedersinken, ein Drängen in die horizontale Lagerung, gegen das schon die aufrechte Haltung, die uns als >homo erectus` auszeichnet, eine fast zu große Anstrengung bedeutet. Mensch zu sein bedeutet, sich aufrecht halten, was eine Spannung und Anstrengung erfordert, doch darin auch eine Selbstmächtigkeit ausdrückt.«(Böhme 2018: 41 [Herv. i. O.])

Gleichzeitig entspricht die zweite Achse dem Zustand der Spannung im Gegensatz zum Zustand der Entspannung:

»Aus der älteren Nerven- und Muskelforschung ist der Zustand der Spannung derjenige, aus dem heraus mentale Aufmerksamkeit und physische Arbeit erfolgt. Spannung heißt, voller Antrieb zu sein und über bereite Kraft zu verfügen, ja einen `drives, eine Spannkraft zur Tätigkeit zu spüren. Der ganze Mensch `spanntく, so 
wie - bei de Sade - der Phallus >spannt<, also >potentia<, >dynamis zeigt, die zur Aktualisierung drängt. Diese Aktualisierung demonstriert Energie. Die ১Spannungく kann vormodern der Metaphorik des gespannten Bogens entnommen sein, von dem der Handlungspfeil auf ein Ziel hin schnellt. Oder, mechanistisch gesehen, wird die Spannung der Feder zur Quelle der gespannten Kraft, aus der wie beim Uhrwerk kontinuierlich ablaufende Bewegungsarbeit gewonnen werden kann.« (a.a.O.: 42 [Herv. i. O.])

Beide Achsen verdeutlichen die Anforderung an den Einzelnen und an die Gesellschaft, sich fortschreitend zu entwickeln, was ihm die wirtschaftlichen Parameter, etwa das Bruttoinlandsprodukt, immer vor Augen halten. Daraus erwachsen kollektive Kulturpathologien einer Gesellschaft, die mit ihren eigenen (Miss-)Erfolgen konfrontiert ist, wie etwa mit der reproduktiven Erschöpfung (sinkende Geburtsraten), der Sinnerschöpfung (Unfähigkeit, die kollektiven Werte, aus denen liberale Gesellschaften leben, zu schöpfen) oder der Erschöpfung politischer Kompetenz (der gesellschaftliche Selbststeuerungsmechanismus erschöpft sich durch die Unfähigkeit der Eliten, die Gesellschaft zu regieren) usw. (vgl. Safranski 2011: 26:05 min.). Bereits Jean-Jacques Rousseau wies darauf hin, dass der gesellschaftliche Fortschritt seinen kollektiven Preis erfordert - die Denaturierung des Menschen, welche durch den technisch-industriellen Fortschritt des Bürgerturms und seine großstädtisch-hektische Lebensform angekurbelt wird (vgl. Böhme 2018: 29) und zu kollektiven Neurosen führt, wie etwa zur Neurasthenie, die »vielleicht weniger eine wirkliche Krankheit [ist], als der genau rechtzeitig erfundene Spiegel, worin die triumphierende Arbeitsgesellschaft ihr erschöpftes Antlitz studieren konnte.« (a.a.O.: 44)

Die Menschheit als solche leidet auch auf globaler Ebene. Spätestens seit dem Ende des Zweiten Weltkrieges und dem Anfang globaler Krisen wie Umweltverschmutzung, Terrorismus, Migration oder lokalen, nicht von Großmächten lösbaren Konflikten, spürt die Menschheit das Leiden, das sie selbst herbeigeführt und nur in einer Kooperation wieder aufheben kann. Die globale Müdigkeit fordert die Menschheit dazu, ein gemeinsames Leben in einer Synchronwelt zu meistern, d.h. in einer Welt, wo die Völker und Gesellschaften zum ersten Mal gleichzeitig ihre Geschichten leben und nicht mehr getrennt von anderen funktionieren können (vgl. Sloterdijk 2011: 46:01 min.). Diese neue Anforderung braucht neue Regeln, die mühsam erarbeitet werden, wovon bspw. das häufige Scheitern des Sicherheitsrats der Vereinten Nationen oder der Umweltschutzabkommen zeugen. Das Müde-Werden durch die Impotenz globaler Aktionsfähigkeit führt zur Mobilisierung Einzelner, die, wie die pakistanische Aktivistin Malala Yousafzai, die schwedische Umweltschützerin Greta Thunberg, der kongolesische Gynäkologe Denis Mukengere Mukwege oder der derzeitige Papst Franziskus, zum Gewissen und zur Stimme der Menschheit werden und sich von dem globalen Leiden berühren lassen. 